EPJ Web of Conferences 38, 07001 (2012)

DOI: $10.1051 /$ epjconf/20123807001

(C) Owned by the authors, published by EDP Sciences, 2012

\title{
Collective spectra along the fission barrier
}

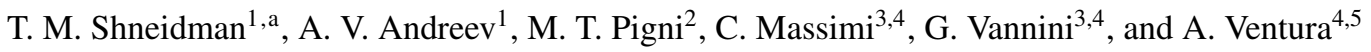 \\ 1 Bogoliubov Laboratory of Theoretical Physics, Joint Institute for Nuclear Research, 141980 Dubna, Russia \\ 2 Nuclear Data Criticality Safety, Oak Ridge National Laboratory, Oak Ridge, TN 37831, USA \\ 3 Dipartimento di Fisica dell' Università di Bologna, 40126 Bologna, Italy \\ 4 INFN, Sezione di Bologna, 40126 Bologna, Italy \\ 5 ENEA, Centro Ricerche Ezio Clementel, 40129 Bologna, Italy
}

\begin{abstract}
Discrete and continuous spectra of fissioning nuclei at the humps of fission barriers (Bohr transition states) and in the intermediate wells (superdeformed and hyperdeformed states) play a key role in the calculation of fission cross sections. A theoretical evaluation of the collective parts of the spectra is possible within the framework of the dinuclear system model, which treats the wave function of the fissioning nucleus as a superposition of a mononucleus configuration and two-cluster configurations in a dynamical way, permitting exchange of upper-shell nucleons between clusters. The impact of theoretical spectra on neutron-induced fission cross sections and, in combination with an improved version of the scission-point model, on angular distribution of fission fragments is evaluated for plutonium isotopes of interest to nuclear energy applications.
\end{abstract}

\section{Introduction}

In the statistical model of nuclear reactions, fission cross sections depend on penetrabilities of potential barriers felt by fissioning nuclei in their ground state or in an excited state. Evaluating barrier parameters and nuclear excitations along the fission barrier so as to reproduce experimental cross sections is a long-standing problem in fission theory: as far as barrier parameters are concerned, theoretical approaches can be divided into two main classes: macroscopic-microscopic methods, originated from Strutinsky's shell and pairing corrections to liquid drop energies [1] and fully microscopic density functional theories, either non relativistic, such as Ref. [2], or relativistic, as Ref. [3]. All of them have reached a high degree of sophistication, by allowing the fissioning nucleus to take a triaxial equilibrium shape at the inner peak of a two-humped barrier and a reflection-asymmetric shape at the outer peak, bringing in this way theoretical values of barrier heights closer to empirical ones. Computing fission cross sections, however, requires also a consistent evaluation of discrete and continuous nuclear spectra at the peaks and in the wells of the barrier: this is relatively easy for non-collective excitations, as discussed in Ref. [2] and references therein for a non-relativistic self-consistent approach, but it is more demanding from a computational viewpoint for collective excitations, since it requires going beyond the mean-field approximation (see Ref. [4] for a relativistic approach). Therefore, we think it worth examining a macroscopic collective model, the dinuclear system (DNS) model, able to predict collective spectra at large deformations, as a possible integration of either micro-macroscopic, or fully microscopic approaches to fission barriers and fission cross sections.

The paper is organized as follows. In Section 2 the DNS model is introduced, the main degrees of freedom

\footnotetext{
a e-mail: shneyd@theor.jinr.ru
}

of the model and the methods of calculation of potential and kinetic energy are discussed. The results of the calculations: fission barrier of $\mathrm{Pu}$ isotopes, the collective spectra in the wells and at the humps of the fission barrier and their impact on calculations of fission cross sections are presented in Section 3. Section 4 introduces the scission point model (SPM) for prediction of mass and charge yields of fission fragments and, in combination with the DNS model, of their angular distributions. Finally, Section 5 is dedicated to conclusions and perspectives.

\section{The Dinuclear System Model}

\subsection{Cluster approach to fission}

Under the expression dinuclear system we understand the system of two touching fragments $\left(A_{1}, Z_{1}\right)$ and $\left(A_{2}, Z_{2}\right)$ with $A_{1}+A_{2}=A$ and $Z_{1}+Z_{2}=Z$ kept together by the moleculartype nucleus-nucleus potential (see Sec. 2.2). The special case of the dinuclear system in which one fragment has zero mass is denoted as a mononucleus.

Each dinuclear system configuration can be specified by the mass-asymmetry $\xi=2 A_{2} / A$, charge-asymmetry $\xi_{Z}=2 Z_{2} / Z$ and the vector of the relative distance $\mathbf{R}=$ $\left(R, \theta_{R}, \phi_{R}\right)$. The values $\xi=0$ or $\xi=2$ correspond to the mononucleus configurations $\left(A_{1}=A, A_{2}=0\right)$ or $\left(A_{1}=\right.$ $\left.0, A_{2}=A\right)$, respectively. Each fragment of DNS is characterized by the quadrupole deformation parameters $\left(\beta_{i}, \gamma_{i}\right)$, $(i=1,2)$ and the set of Euler angles $\Omega_{i}=\left(\phi_{i}, \theta_{i}, \alpha_{i}\right)$, $(i=1,2)$ describing orientation of the fragments in the laboratory frame. These give a total of 15 degrees of freedom, which can be used to describe the collective motion in a dinuclear system consisting of quadrupole-deformed fragments. In the low energy limit the adiabatic approximation seems to be justified; thus, single-particle excitations of fragments are neglected.

This is an Open Access article distributed under the terms of the Creative Commons Attribution License 2.0, which permits unrestricted use, distribution, and reproduction in any medium, provided the original work is properly cited. 
The main idea of the DNS model is that neither a single mononucleus nor a single dinuclear system can alone describe the nucleus. The intrinsic nuclear wave function can rather be described as a superposition of the mononucleus and different dinuclear configurations. The mononucleus is taken to be quadrupole-deformed; thus, in the DNS model, the contribution to the reflection asymmetric deformation of the nucleus is connected with the nonzero weight of the asymmetric dinuclear systems. This choice of the nuclear wave function gives us the possibility of describing in one approach the ground state properties of heavy nuclei and the fission process as well. The various stages of fission can be characterized by the major contributions of different dinuclear systems to the total wave function of nucleus.

\subsection{Potential energy calculations}

On the assumption of a small overlap of nuclei in the dinuclear system, the potential energy of the DNS model is calculated as the sum of the binding energies of the fragments $B_{i},(i=1,2)$ of the dinuclear system and the interaction $V^{\text {int }}$ between the fragments [5]

$$
\begin{aligned}
& U\left(\xi, \xi_{Z}, \beta_{i}, \gamma_{i}, \Omega_{i}, R_{i},\{i=1,2\}\right) \\
& =B_{1}\left(A_{1}, Z_{1}, \beta_{1}, \gamma_{1}\right)+B_{2}\left(A_{2}, Z_{2}, \beta_{2}, \gamma_{2}\right) \\
& +U^{\text {int }}\left(\xi, \xi_{Z}, R, \beta_{i}, \gamma_{i}, \Omega_{i}, R_{i},\{i=1,2\}\right)
\end{aligned}
$$

This approach allows us to calculate the potential energy as a function of the DNS degrees of freedom: mass and charge asymmetries $\xi$ and $\xi_{Z}$, internuclear distance $R$, deformation parameters $\left(\beta_{i}, \gamma_{i}\right)$ and orientation angles $\Omega_{i}$ of the fragments $(i=1,2)$.

The binding energy of the fragment $\left(A_{i}, Z_{i}\right)$ with deformation parameters $\left(\beta_{i}, \gamma_{i}\right)$ is calculated as the sum of the liquid drop energy $U_{i}^{L D}$ and the shell correction term $\delta U_{i}^{\text {shell }}$

$$
B_{i}=U_{i}^{L D}+\delta U_{i}^{\text {shell }}, \quad(i=1,2) .
$$

The liquid drop energy is calculated using the parameters that give, together with shell corrections, the best fit to the experimental binding energies of the separate fragments at their ground state deformations [6]. The deformationdependent shell corrections are calculated with the twocenter shell model (TCSHM) [7].

The version of the TCSHM we used limits the variety of fragment equilibrium shapes to the axially-symmetric ones (i.e. $\gamma_{1}=\gamma_{2}=0$ ). This limitation may lead to overestimated heights of fission barrier peaks (see Sec. 3.3). This drawback, however, can be avoided in future by using a more refined shell model. This modification will not affect much the collective spectra along the fission barrier.

The interaction energy, $U^{\text {int }}$, is calculated as a sum of the Coulomb $U^{C}$ and nuclear $U^{N}$ interactions. The latter is taken in the form of a double folding of nuclear densities and density-dependent Skyrme-type nucleon-nucleon forces [8].

The nuclear part of the interaction energy is attractive for $R>R_{1}+R_{2}-a$ and repulsive for $R<R_{1}+R_{2}-a$, where $a \approx 1.5 \mathrm{fm}$, thus simulating the Pauli principle and the structure forbiddenness effects in the motion to smaller values of $R$. As a result of the interplay between nuclear and Coulomb interaction, the interaction potential $U^{i n t}$ can have a potential pocket corresponding to the pole-to-pole configuration of nearly touching fragments. The depth and the position of this pocket depends on the masses and deformations of the fragments of the dinuclear system. For asymmetric dinuclear systems corresponding to actinides the minimum of the potential pocket is located at the touching distance. For nearly symmetric dinuclear systems, depending on fragment deformations, the minimum of $U^{\text {int }}$ is shifted to distances $(0.5-1)$ fm larger than the touching distance. The existence of the minimum of the potential energy of the dinuclear system at distances equal or larger than the touching one justifies the expression (1) for the potential energy used in the DNS model.

In order to calculate a fission barrier, the potential energy of the DNS model has to be minimized with respect to all degrees of freedom at a given value of the mass quadrupole moment of the system. The problem is simplified since we can consider only dinuclear systems in the minimum of the potential pocket $\left(R=R_{m}\right)$ with axiallydeformed fragments in pole-to-pole orientation $\left(\Omega_{1}=\Omega_{2}=\right.$ $0)$. Moreover, the potential energy of the DNS model as a function of charge asymmetry has a deep minimum corresponding to $N / Z$-equilibrium. Thus, for a given mass asymmetry $\xi$ the charge asymmetry is fixed at $\xi_{Z}=\xi_{Z}^{(e q)}(\xi)$.

\subsection{Kinetic Energy of the DNS model}

In order to calculate the collective spectra in the intermediate wells and at the humps of the fission barrier, the Hamiltonian of the DNS model must be formulated and diagonalized. The classical expression for the kinetic energy is obtained by considering the model system of two fragments with masses $A_{1} m_{0}$ and $A_{2} m_{0}$, where $m_{0}$ is the nucleon mass. The total mass of the system, $A m_{0}$, is fixed $\left(A_{1}+A_{2}=A\right)$. Due to nucleon transfer, the masses of the fragments change in time, leading to the motion in mass asymmetry $\xi$. Since potential energy calculations strongly support the $N / Z$ equilibrium along the fission barrier, charge asymmetry is not considered as an independent variable. In order to avoid the consideration of the center of mass motion of the whole system, the origin of the laboratory frame is placed in the center of mass of the whole nuclear system. The classical expression for the kinetic energy can be written as a sum of terms describing motion in mass asymmetry, relative motion of the fragments, and kinetic energies $T_{\text {intr }}^{(i)}(i=1,2)$ of intrinsic motion of fragments

$$
T=\frac{1}{2} B_{\xi} \dot{\xi}^{2}+\frac{1}{2} \mu \dot{\mathbf{R}}^{2}+T_{\text {intr }}^{(1)}+T_{\text {intr }}^{(2)}
$$

In the above-given expression $\mu=m_{0}\left(A_{1} A_{2}\right) / A$ is the reduced mass of the system, and $B_{\xi}$ is the effective mass for the mass asymmetry motion.

Treating the intrinsic motions of the fragments as quadrupole s urface motions we can use the results of the rotational-vibrational model [9] and write $(i=1,2)$

$$
T_{\text {intr }}^{(i)}=\frac{1}{2} D_{i}(\xi)\left(\dot{\beta}_{i}^{2}+\beta_{i}^{2} \dot{\gamma}_{i}^{2}\right)+\frac{1}{2} \sum_{k} \omega_{k}^{2} \mathfrak{J}_{k}^{(i)}\left(\beta_{i}, \gamma_{i}\right),
$$

where the moments of inertia are

$$
\mathfrak{J}_{\kappa}^{(i)}=4 D_{i}(\xi) \beta_{i}^{2} \sin ^{2}\left(\gamma_{i}-\frac{2 \pi \kappa}{3}\right), \quad(\kappa=1,2,3) .
$$


In the above-given expressions $\beta_{i}$ and $\gamma_{i}$ are the deformation parameters of the fragments and $D_{i}(\xi)(i=1,2)$ are the effective masses for the quadrupole vibrations of the fragments, treated as parameters of the model.

If we assume that the DNS potential energy has a local minimum at $\xi=\xi_{0}$, the motion in mass asymmetry can be treated as a vibration around $\xi_{0}$. The mass parameters involved in the expressions $(3,4,5)$ can then be replaced by their values at $\xi=\xi_{0}$

$$
D_{i}(\xi) \approx D_{i}\left(\xi_{0}\right), \quad B_{\xi}(\xi) \approx B_{\xi}\left(\xi_{0}\right)
$$

The collective kinetic energy (3) is quantized according to the usual Pauli quantization prescription. Thus, the quantized kinetic energy can be written as $(\mu(\xi)=A \xi(1-$ $\xi))$

$$
\begin{aligned}
\hat{T} & =-\frac{\hbar^{2}}{2 B_{\xi}} \frac{1}{\mu^{3 / 2}(\xi)} \frac{\partial}{\partial \xi} \mu^{3 / 2}(\xi) \frac{\partial}{\partial \xi}-\frac{\hbar^{2}}{2 \mu(\xi)} \frac{1}{R^{2}} \frac{\partial}{\partial R} R^{2} \frac{\partial}{\partial R} \\
& +\hat{T}_{r o t}+\hat{T}_{i n t r}, \\
\hat{T}_{r o t} & =\frac{\hbar^{2}}{2 \mu(\xi) R^{2}} \hat{L}_{0}^{2}+\sum_{i=1,2}\left\{\frac{\hbar^{2}}{2} \sum_{\kappa=1}^{3} \frac{\hat{L}_{(i), \kappa}^{2}}{\mathfrak{J}_{\kappa}^{(i)}\left(\beta_{i}, \gamma_{i}\right)}\right\} \\
\hat{T}_{\text {intr }} & =\sum_{i=1,2}\left\{-\frac{\hbar^{2}}{2 D_{i}\left(\xi_{0}\right)}\right. \\
& \left.\times\left[\frac{1}{\beta_{i}^{4}} \frac{\partial}{\partial \beta_{i}} \beta_{i}^{4} \frac{\partial}{\partial \beta_{i}}+\frac{1}{\beta_{i}^{2}} \frac{1}{\sin 3 \gamma_{i}} \frac{\partial}{\partial \gamma_{i}} \sin 3 \gamma_{i} \frac{\partial}{\partial \gamma_{i}}\right]\right\}
\end{aligned}
$$

The first term in (7) describes the motion in mass asymmetry, the second term describes relative motion of the clusters, $\hat{T}_{r o t}$ is the kinetic energy of the rotational motion of the fragments and the dinuclear system as a whole, and $\hat{T}_{i n t}$ is the kinetic energy for the intrinsic motion (quadrupole vibrations) of the fragments.

\section{Application of the DNS model to fission}

\subsection{Fission barrier for $\mathrm{Pu}$ isotopes}

According to the discussion in Sect. (2.2), in order to determine the fission barrier, it is enough to consider only mononucleus and dinuclear systems with axially-deformed fragments in $N / Z$-equilibrium placed in the minimum of the potential pocket $\left(R=R_{m}, \Omega_{1}=\Omega_{2}=0\right)$. The potential energy of the DNS model is minimized with respect to mass-asymmetry $\xi$ and deformation parameters $\beta_{1}$ and $\beta_{2}$ of the fragments. The minimization is performed for each fixed value of the mass quadrupole moment, which is expressed in Ref. [10] by

$$
Q^{(m)}=2 m_{0} \frac{A_{1} A_{2}}{A} R_{m}^{2}+Q_{1}^{(m)}\left(\beta_{1}\right)+Q_{2}^{(m)}\left(\beta_{2}\right)
$$

where $Q_{i}^{(m)}\left(\beta_{i}\right),(i=1,2)$ are the mass quadrupole moments of the fragments.

The model predicts for ${ }^{240} \mathrm{Pu}$ a three-humped barrier with parameters (in $\mathrm{MeV}$ ) $V_{A}=5.27, V_{I I}=3.09, V_{B}=6.30$, $V_{I I I}=2.65, V_{C}=3.30$. Here, the first, second, and third saddle points are denoted as $A, B$, and $C$, respectively. The superdeformed (SD) and hyperdeformed (HD) minima are denoted as $I I$ and $I I I$.
Our calculations show that the ground state and SD minima of the fission barrier correspond to the mononucleus configuration taken at deformations $\beta_{2}=0.3$ and $\beta_{2}=$ 0.64 , respectively. The only dinuclear system which can compete with the mononucleus in the vicinity of these minima is the dinuclear system with an alpha-particle as the light cluster, ${ }^{A-4}(Z-2)+{ }^{4} \mathrm{He}$. The energy of the alphaparticle dinuclear system is about $1.5 \mathrm{MeV}$ larger than the energy of the mononucleus taken at the same quadrupole moment near the ground state or the SD minimum.

When the system approaches hump "A" between ground state and SD minimum, or hump "B" between SD and HD minima the situation changes. The potential energies of the alpha-particle dinuclear system and of the mononucleus are, again, much lower than the energies of other configurations. In these cases, however, the competition ends in favour of the alpha-particle dinuclear system, since its potential energy is smaller than the energy of the mononucleus by about $0.5 \mathrm{MeV}$. Thus at the first and the second hump of the fission barrier the fissioning nucleus has stable reflection-asymmetric deformation. This is at variance with other models, which predict a reflection-asymmetric shape only at the outer hump.

When we move along the fission barrier beyond the second hump, the mass-asymmetry starts to increase rapidly. The HD minimum and the third hump correspond to ${ }^{82} \mathrm{Ge}+{ }^{158} \mathrm{Sm}$ (with $\mathfrak{J}=307 \hbar^{2} / \mathrm{MeV}$ ) and ${ }^{90} \mathrm{Kr}+{ }^{150} \mathrm{Ce}$ systems, respectively. Owing to the modest height of the third peak, states in the third well (hyperdeformed states) would have too short a life time to be observed in a fission reaction.

The calculation have been performed also for ${ }^{239} \mathrm{Pu}$ and ${ }^{238} \mathrm{Pu}$ isotopes. The calculated fission barriers have in the three cases the first peak smaller than the second peak, at variance with the empirical barriers, as shown in Sect. 3.3.

\subsection{Collective spectra along the fission barrier}

In principle, the collective spectrum of the DNS model is obtained by diagonalization of the Hamiltonian depending on all 15 degrees of freedom described in the previous Section. In practice, however, we are interested only in the collective degrees of freedom that produce the rotational bands starting below the first few-quasiparticle states. At higher energies the use of a level density formalism is more appropriate. In the description of the collective excitations up to the second saddle point the relevant degrees of freedom are related to the motion in mass asymmetry, rotation of the DNS as a whole, and rotation and quadrupole vibrations of the heavy fragment of the DNS. Since the main contribution to the wave function comes from mononucleus and alpha-particle DNS the intrinsic excitations of the spherical light fragment are neglected. The form of the simplified Hamiltonian and details of the calculations are presented in [11], where the model is applied to the description of the excitation spectra of different actinides at normal deformation.

The results of the calculation of the collective spectra of ${ }^{240} \mathrm{Pu}$ at superdeformation are shown in figure 1 together with available experimental data. One can see that the overall agreement between the calculated and experimental collective spectra is rather good. 

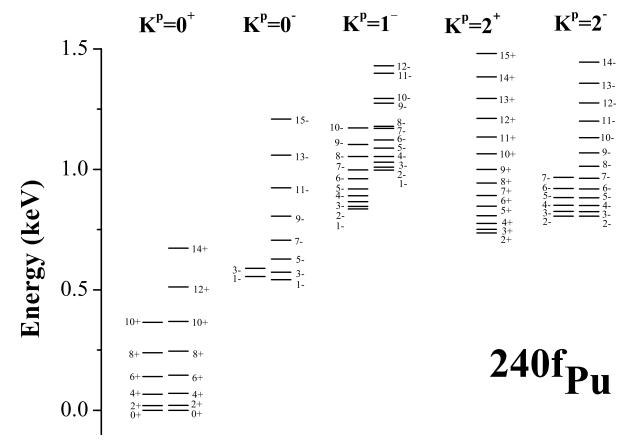

Fig. 1. Calculated and experimental low-energy collective spectrum of ${ }^{240} \mathrm{Pu}$ in the superdeformed well. Experimental energies, spin and parity assignments are taken from [12].

The calculated level scheme is presented as a sequence of positive- and negative-parity rotational bands. Due to the coupling between rotational and vibrational degrees of freedom, the third projection of angular momentum $K$ is not a good quantum number. However, $K$ can still be used to interpret the results of the calculations. The first $K^{p}=0^{+}$and $K^{p}=2^{+}$rotational bands are related, respectively, to the excitation of beta- and gamma-vibrations of the heavy fragment. The second $K^{p}=0^{+}$rotational band is built on the excitation in the mass-asymmetry degree of freedom. Each positive-parity band is accompanied by a series of negative-parity bands, which appear owing to the non-zero weight of the asymmetric dinuclear systems producing the reflection-asymmetric deformation. Since the contribution from the asymmetric DNS to the nuclear wave function is very small (about 2-3\%) the reflectionasymmetric deformation is not stable and the negativeparity states are shifted upward with respect to the corresponding positive-parity states.

The situation changes when we consider the collective bands of the system at the saddle points. Here, the potential energy of the alpha-particle DNS is smaller than the energy of the mononucleus. As a result the weight of asymmetric dinuclear systems is increased (about $15 \%$ ). Our calculation shows that such an increase is already enough to stabilize the reflection-asymmetric deformation. This is clearly seen from the spectrum of ${ }^{240} \mathrm{Pu}$ at the second saddle point (figure 2). The ground state band now consists of states with all angular momenta $L$ and parities $p=(-1)^{L}$. For $K \neq 0$ the states with the same angular momentum $L$ and different parities are degenerate. Such a degeneracy does not appear in the spectrum at ground state and superdeformation owing to the larger contribution of the mononucleus configuration.

The collective spectra at the first $(i=1)$ and the second $(i=2)$ saddle points are strongly relevant to fission cross section calculations. The results of the calculation of the collective spectra of ${ }^{240} \mathrm{Pu}$ at the different saddle points can be very accurately approximated as

$$
\begin{aligned}
& E\left(L, K, p, n_{b}, n_{\gamma}\right)=E_{f i} \\
& +\epsilon_{i}\left(K, v, p, n_{b}, n_{\gamma}, n_{\text {m.a. }}\right)+\frac{\hbar^{2}}{2 \mathfrak{J}_{i}}\left(L(L+1)-K^{2}\right), \\
& L^{p}=0^{+}, 1^{-}, 2^{+}, 3^{-}, \ldots, K=0, \\
& L^{p}=K^{ \pm},(K+1)^{ \pm},(K+2)^{ \pm}, \ldots, K \neq 0,
\end{aligned}
$$

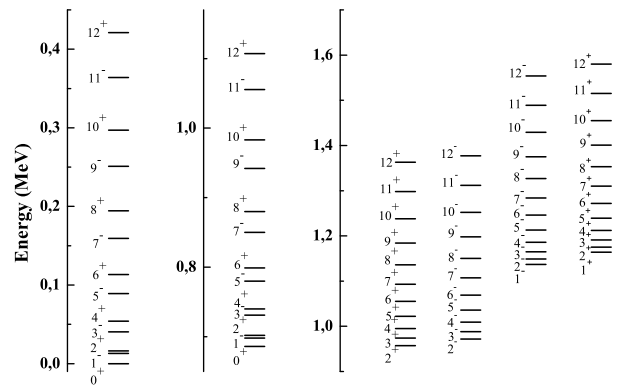

Fig. 2. The collective spectrum of ${ }^{240} \mathrm{Pu}$ at the second saddle point.

where $E_{f i}$ are the heights of the saddle points, $\epsilon_{i}\left(K, v, p, n_{b}\right.$, $\left.n_{\gamma}, n_{\text {m.a. }}\right)$ are the bandheads and $\mathfrak{J}_{i}$ are the moments of inertia. The bandheads can be written in the form

$$
\begin{aligned}
& \epsilon_{i}\left(K, v, p, n_{b}, n_{\gamma}, n_{m . a .}\right)=\hbar \omega_{b}^{(i)} n_{m . a .} \\
& +\hbar \omega_{b}^{(i)}\left(2 n_{b}+|K-v|\right)+\hbar \omega_{\gamma}^{(i)}\left(2 n_{\gamma}+v\right) \\
& v=0,2, \ldots, K, \quad K=0,1,2 \ldots
\end{aligned}
$$

In Formula (10), $\hbar \omega_{m . a}^{(i)}$ is the frequency of the vibrations in mass asymmetry degree of freedom, $\hbar \omega_{b}^{(i)}$ is the frequency of the small angular oscillations of the dinuclear system around the pole-to-pole configuration, $\hbar \omega_{\gamma}^{(i)}$ is the frequency of the gamma-vibrations of the ${ }^{236} \mathrm{U}$ nucleus. At the saddle point, the beta band is missing because it is a vibration in the fission direction. The values of $\hbar \omega_{\text {m.a. }}, \hbar \omega_{b}$, $\hbar \omega_{\gamma}$, and $\hbar^{2} / 2 \mathfrak{J}$ at the different saddle points of ${ }^{240} \mathrm{Pu}$ are presented in Table 1.

Table 1. Bandhead energies and rotational constants $\hbar^{2} / 2 \mathfrak{J}$ at saddle points "A" and "B" of ${ }^{240} \mathrm{Pu}$ (in $\mathrm{MeV}$ ).

\begin{tabular}{cllll}
\hline Saddle Point & $\hbar \omega_{b}$ & $\hbar \omega_{\text {m.a. }}$ & $\hbar \omega_{\gamma}$ & $\hbar^{2} / 2 \mathfrak{J}$ \\
\hline "A" & 0.841 & 0.616 & 1.136 & 0.0037 \\
"B" & 1.137 & 0.686 & 0.957 & 0.0027 \\
\hline
\end{tabular}

\subsection{Calculation of fission cross sections}

While spectroscopy in the ground state and isomeric well of ${ }^{240} \mathrm{Pu}$ permits a direct comparison of the collective spectra computed by means of the DNS with experimental levels and related electromagnetic transitions, no such possibility exists for discrete levels predicted at the humps of the fission barrier, but an indirect test of their reliability is provided by their identification with the Bohr transition states acting as doorways to fission at low excitation energies.

In the statistical model of nuclear rections, fission is a compound nucleus process whose cross section is the product of a compound nucleus formation cross section, $\sigma_{c n}\left(E^{*}, J, p\right)$, times a fission probability, $P^{f}\left(E^{*}, J, p\right)$, depending on the excitation energy, $E^{*}=E_{n}+B_{n}$, of the compound nucleus, with $E_{n}$ the incident neutron energy and $B_{n}$ its binding energy in the compound nucleus, and on the angular momentum, $J$, and parity, $p$ of the compound 
nucleus; $P^{f}\left(E^{*}, J, p\right)$ can be written as the ratio of the transmission coefficient of the fission channel, $T_{f}\left(E^{*}, J, p\right)$, to the sum of the transmission coefficients of all competing reaction channels at the given excitation energy of the compound nucleus

$$
\sigma_{n f}\left(E_{n}\right)=\sum_{J^{p}} \sigma_{c n}\left(E^{*}, J^{p}\right) \frac{T_{f}\left(E^{*}, J^{p}\right)}{\sum_{c} T_{c}\left(E^{*}, J^{p}\right)} .
$$

Fission transmission coefficients are usually evaluated by solving the Schrödinger equation with a multiple-humped potential in the fission coordinate, $\beta$, approximated by a set of smoothly joined parabolas

$$
V_{j}(\beta)=V_{j}^{0}+\frac{(-1)^{j}}{2} \mu\left(\hbar \omega_{j}\right)^{2}\left(\beta-\beta_{j}\right)^{2}, j=1, \ldots, 2 N_{h}-1 .
$$

Here, $N_{h}$ is the number of humps of the barrier, $\mu$ is an inertial mass parameter approximated by the semiempirical expression $\mu=0.054 A^{5 / 3} \mathrm{MeV}^{-1}$, while heights $V_{j}^{0}$ and curvatures $\hbar \omega_{j}$ are usually adjusted so as to reproduce experimental fission cross sections. For a single-humped barrier, $V(\beta)=V_{0}+\frac{\mu}{2}(\hbar \omega)^{2} \beta^{2}$, the transmission coefficient is given by the well-known Hill-Wheeler formula, $T_{H W}\left(E^{*}\right)=1 /\left(1+\exp \left[2 \pi \frac{V_{0}-E^{*}}{\hbar \omega}\right]\right)$. According to the Bohr hypothesis, discrete transition states are arranged in rotational bands based on each barrier hump: in case of axial symmetry of the sistem, bands can be labeled with bandhead energy $\varepsilon$, projection $K$ of the total angular momentum on the nuclear symmetry axis and parity $p$. Discrete spectra evolve into a continuum described by a level density, $\rho(\epsilon, I, p)$, above a cutoff energy, $E_{c}$, which for even-even nuclei is commonly taken to coincide with the lowest twoquasiparticle excitation.

For multiple-humped barriers the transmission coefficients have expressions more complicated than the HillWheeler formula, worked out in full detail in JWKB approximation in Ref. [13] for two- and three-humped barriers, including also the effect of imaginary potential components in intermediate wells: this is the optical model of fission, simulating the damping of collective states in the intermediate wells due to their coupling with compound nucleus states at normal deformation. The optical model of fission is part of the nuclear reaction models (optical, statistical, pre-equilibrium...) on which the Empire system of codes [14] is based. In the present work, the cross sections of neutron-induced fission of ${ }^{239} \mathrm{Pu}$, yielding ${ }^{240} \mathrm{Pu}$ as the fissioning compound nucleus, and of the competing reaction channels are computed by means of a modified version of Empire (Rivoli). In addition to discrete spectra at normal deformation, superdeformation and hyperdeformation, corresponding to ground state, second and third well of the fission barrier, the DNS model yields transition states at the intermediate peaks. Here, the favoured nuclear shape turns out to be reflection asymmetric, due in the present case to the increased influence of the ${ }^{4} \mathrm{He}^{236} \mathrm{U}$ configuration with respect to the ${ }^{240} \mathrm{Pu}$ mononucleus configuration: in addition to barrier parameters evaluated in the macroscopicmicroscopic approach described in Sect.3, discrete transition states computed by the DNS model and continuum states described by a level density formalism based on the superfluid generalized model contained in Empire, with an energy dependent collective enhancement factor appropriate to reflection asymmetric shapes, are basic ingredients of the fission input of the code. While theoretical collective spectra are kept fixed in our calculations, we take the liberty of adjusting barrier heights on fission data, since we know that they may be overestimated in the present version of the macroscopic-microscopic model, which does not allow for triaxial equilibrium shapes along the barrier.

In order to compute the fission cross section up to $E_{n}$ $=20 \mathrm{MeV}$, barrier parameters and collective spectra are calculated not only for ${ }^{240} \mathrm{Pu}$, the compound nucleus in the first-chance fission reaction $(n, f)$, but also for ${ }^{239} \mathrm{Pu}$, compound nucleus in the second-chance fission $\left(n, n^{\prime} f\right)$ and ${ }^{238} \mathrm{Pu}$, compound nucleus in the third-chance fission $(n, 2 n f)$. In order to reduce the degree of arbitrariness in adjusting barrier parameters, we choose the barrier parameters of ${ }^{239} \mathrm{Pu}$ so as to reproduce the ${ }^{238} \mathrm{Pu}(n, f)$ cross section and adjust those of ${ }^{238} \mathrm{Pu}$ on the ${ }^{237} \mathrm{Pu}(n, f)$ cross section, as shown in figure 3.

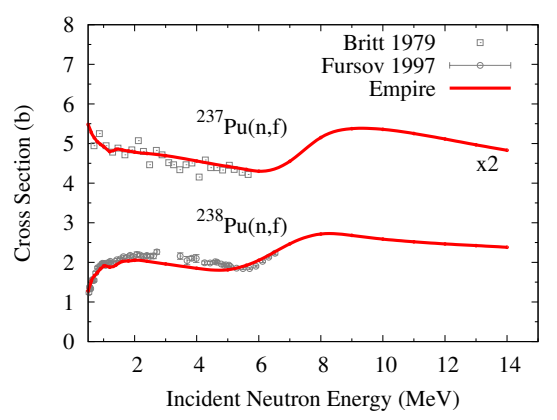

Fig. 3. Fission cross sections of ${ }^{237} \mathrm{Pu}$ and ${ }^{238} \mathrm{Pu}$. Red curves: Empire 3.1 calculations; experimental data: ${ }^{237} \mathrm{Pu}$ : Britt 1979, Ref. [15]; ${ }^{238}$ Pu: Fursov 1997: Ref. [16].

The final result for the neutron-induced fission cross section of ${ }^{239} \mathrm{Pu}$ is shown in figure 4 , in comparison with selected experimental data.

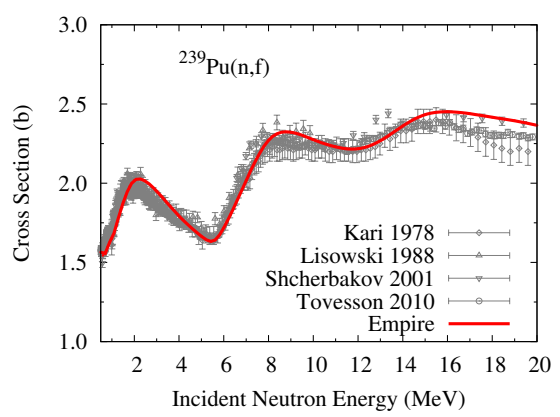

Fig. 4. Fission cross section of ${ }^{239} \mathrm{Pu}$. Red curve: Empire $3.1 \mathrm{cal}-$ culation; experimental data: Refs.[17-20].

The adopted values of barrier heights and curvatures are given in Table 2; here, letter $A$ refers to the inner hump, letter $I$ to the isomeric well and letter $B$ to the outer hump. Not surprisingly, the adopted barrier parameters are close to the empirical values in the literature [21], with the heights of the two peaks exchanged with respect to theoretical predictions.

Summing up, a reasonable fit of experimental data is obtained at the price of modifying theoretical barrier pa- 
Table 2. Fission barrier parameters of ${ }^{238,239,240} \mathrm{Pu}$ (in $\mathrm{MeV}$ ). The values calculated with DNS model are given in brackets.

\begin{tabular}{cllllll}
\hline Comp. nucleus & $V_{A}$ & $\hbar \omega_{A}$ & $V_{I}$ & $\hbar \omega_{I}$ & $V_{B}$ & $\hbar \omega_{B}$ \\
\hline${ }^{238} \mathrm{Pu}$ & 5.93 & 0.99 & 2.00 & 0.50 & 4.63 & 0.60 \\
& $(4.9)$ & & $(3.3)$ & & $(6.4)$ & \\
${ }^{239} \mathrm{Pu}$ & 6.25 & 0.92 & 2.20 & 0.50 & 5.66 & 0.50 \\
& $(5.2)$ & & $(3.2)$ & & $(5.9)$ & \\
${ }^{240} \mathrm{Pu}$ & 5.74 & 0.94 & 2.0 & 0.50 & 4.85 & 0.65 \\
& $(5.3)$ & & $(3.1)$ & & $(6.3)$ & \\
\hline
\end{tabular}

rameters, while theoretical discrete spectra are kept in the wells and on the humps of the barrier: in the latter case, the assumption of reflection-asymmetric shapes at all excitation energies appears to be compatible with experimental data.

\section{Scission point model and applications}

As shown in our calculations, after passing the second saddle point the fissioning nucleus begins to develop in mass asymmetry degree of freedom. Simultaneously, the dinuclear system characterized by the mass asymmetry $\xi$ has the possibility of decaying in relative distance coordinate $R$ and undergoing fission. The competition between these two processes determines the dynamics of the fissioning nucleus beyond the saddle point.

As a first step towards the solution of these problems, in this work we consider the limiting case of a well defined scission configuration, assuming that statistical equilibrium among various degrees of freedom of the system is established before the decay in $R$ [6]. It is thus possible to calculate the probabilities of different scission point configurations, and, consequently, to describe various fission characteristics, such as mass and charge yields as well as kinetic-energy and angular distributions of fission fragments.

We assume that the fissioning nucleus at the scission point forms a dinuclear system with the two fragments in contact. The scission point is at nuclear distance $R=R_{b}$, with $R_{b}$ the position of the outer barrier in relative distance between the fragments. The relative probability of formation of a system of two fragments with mass and charge numbers $\left(A_{1}, Z_{1}\right)$ and $\left(A_{2}, Z_{2}\right)$ and deformations $\beta_{1}$ and $\beta_{2}$ can be written as

$$
P\left(\left\{A_{i}, Z_{i}, \beta_{i}\right\}, R_{b}\right)=P_{0} \exp \left(-\frac{U\left(\left\{A_{i}, Z_{i}, \beta_{i}\right\}, R_{b}\right)}{T}\right),
$$

where $T$ is the temperature related to the excitation energy $T=\left(E^{*} / a\right)^{1 / 2}$ and $a=A / 12 \mathrm{MeV}^{-1}$, with $A$ the mass number of the fissioning nucleus. The potential energy in (13) is calculated by means of Eq. (1) for axially-deformed fragments in pole-to-pole configuration. The damping of shell corrections with excitation energy is taken into account in calculations of the binding energies of the fragments.

Using the Boltzmann factor given by formula (13), we can calculate mean values of fragment masses $A_{H}$ and $A_{L}$, with $A_{H}+A_{L}=A$, and corresponding deformations $\beta_{H}$ and $\beta_{L}$. These determine the most favourable dinuclear configuration at the scission point. If the fissioning nucleus at the scission point has total angular momentum $J$ and projection $M$ on the direction of the incident neutron beam, it is possible to determine a set of states with quantum numbers $J$ and $M$ that are related to the excitation of different rotational and vibrational degrees of freedom of the most favourable dinuclear configuration. In particular, the rotational degrees of freedom correspond to the independent rotations of the two fragments and to the rotation of the DNS as a whole.

The Hamiltonian describing the collective excitations of the DNS consisting of fragment $\left(A_{H}, Z_{H}\right)$ with deformation $\beta_{H}$, and fragment $\left(A_{L}, Z_{L}\right)$ with deformation $\beta_{L}$ can be written as:

$$
\begin{aligned}
\hat{H} & =\frac{\hbar^{2}\left(\hat{J}_{H}^{2}-\hat{J}_{H,(3)}^{2}\right)}{2 \mathfrak{J}_{H}^{\perp}}+\frac{\hbar^{2} \hat{J}_{H,(3)}^{2}}{2 \mathfrak{J}_{H}^{\|}} \\
& +\frac{\hbar^{2}\left(\hat{J}_{L}^{2}-\hat{J}_{L,(3)}^{2}\right)}{2 \mathfrak{J}_{L}^{\perp}}+\frac{\hbar^{2} \hat{J}_{L,(3)}^{2}}{2 \mathfrak{J}_{L}^{\|}} \\
& +\frac{\hbar^{2} \hat{J}_{0}^{2}}{2 \mathfrak{J}_{0}}+U\left(\Omega_{1}, \Omega_{2}\right),
\end{aligned}
$$

where $U\left(\Omega_{1}, \Omega_{2}\right)$ is the potential energy of the dinuclear system as a function of the relative orientation of fragments, $\hat{J}_{H}\left(\hat{J}_{H,(3)}\right), \hat{J}_{L}\left(\hat{J}_{L,(3)}\right)$, and $\hat{J}_{0}$ are the angular momenta (and their projections on the DNS symmetry axis) of the two fragments and the relative angular momentum, respectively. The relative moment of inertia of the system is denoted as $\mathfrak{J}_{0}$. The quantities $\mathfrak{J}_{H}^{\perp}\left(\mathfrak{J}_{H}^{\|}\right), \mathfrak{J}_{L}^{\perp}\left(\mathfrak{J}_{L}^{\|}\right)$are the perpedicular (parallel) moments of inertia of the corresponding fragments calculated in the rigid body limit. The choice of rigid body moments of inertia are justified since the dinuclear system at the scission point is highly excited.

Diagonalizing the Hamiltonian (14) we obtain its eigenvalues $E_{J M p}^{\lambda, K_{H}, K_{L}}\left(A^{(H, L)}, Z^{(H, L)}, \beta^{(H, L)}\right)$ as well as its eigenfunctions, $\Psi_{J M p}^{\lambda, K_{H}, K_{L}}\left(\Omega_{H}, \Omega_{L}, \Omega_{0}\right)$.

Assuming that the decay of the scission-point configuration is a fast process, the probability of emitting fission fragments from the state $\Psi^{\lambda}$ at an angle $\theta$ is given by

$$
P_{J M p}^{\lambda, K_{H}, K_{L}}=\sin \theta d \theta \int\left|\Psi_{J M p}^{\lambda, K_{H}, K_{L}}\right|^{2} d \Omega_{H} d \Omega_{L} d \phi
$$

Here, the change of the angular distribution caused by the Coulomb excitation subsequent to the fission is neglected.

The angular distribution of fission fragments for the channel characterized by angular momentum $J$, projection $M$, parity $p$ at temperature $T$ can be written as

$$
W_{J M p}\left(E^{*}, \theta\right)=A(J) \sum_{\lambda, K_{H}, K_{L}} \exp \left[-\frac{E_{J M p}^{\lambda, K_{H}, K_{L}}}{T\left(E^{*}\right)}\right] P_{J M p}^{\lambda, K_{H}, K_{L}},(16)
$$

with $A(J)$ a normalization constant.

Summing over the contributions of different channels, it is possible to compute the total angular distribution, $W(\theta)$; a quantity that is commonly compared with experimental data is the angular anisotropy, $W(\theta=0) / W(\theta=\pi / 2)$, evaluated as a function of $E_{n}$, as shown in figure 5 for ${ }^{239} \mathrm{Pu}(n, f)$.

Our theoretical results are in good agreement with experimental data at low energies $\left(E_{n}<4 \mathrm{MeV}\right)$. The behaviour at higher energies, where the experimental angular 


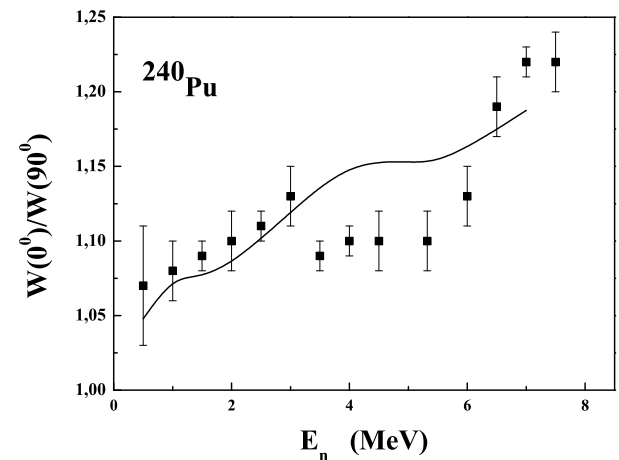

Fig. 5. Calculated and experimental angular anisotropy of fission fragments vs incident neutron energy for ${ }^{239} \mathrm{Pu}(n, f)$. Data are taken from Ref. [22].

anisotropy first decreases and then rapidly increases again above $6 \mathrm{MeV}$ may be due to the interplay of two factors that are not yet included in our model. The decrease of the angular anisotropy may be related to the threshold of excitation of two-quasiparticle states in the fragments of the most favourable dinuclear configuration. The rapid increase above $6 \mathrm{MeV}$ is associated with the threshold of second-chance fission.

The relative mass distribution of fission fragments is obtained in the form

$$
Y\left(A_{1}\right) \sim \sum_{Z_{1}} \int \exp \left[-\frac{U\left(\left\{A_{i}, Z_{i}, \beta_{i}\right\}, R_{b}\right)}{T}\right] \mathrm{d} \beta_{1} \mathrm{~d} \beta_{2} .
$$

figure 6 shows results of calculations of mass distribution for thermal-neutron induced fission of ${ }^{239} \mathrm{Pu}$ in good agreement with experimental data.

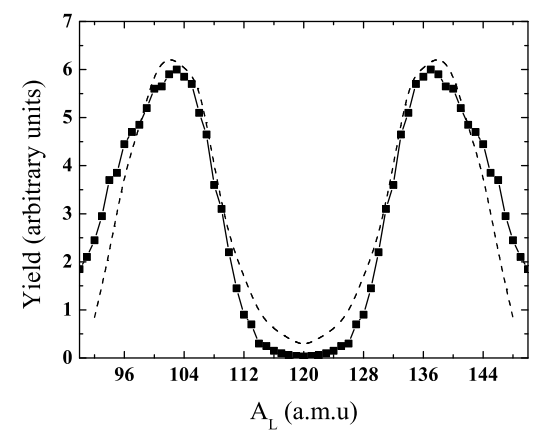

Fig. 6. Fragment mass distribution for thermal-neutron induced fission of ${ }^{239} \mathrm{Pu}$. Dashed line: calculation in the scission point model. Points connected by the solid line as an eye guide: experimental (pre-neutron emission) mass yields given by Ref. [23].

Another observable that can be computed by means of the scission-point model is the average kinetic energy of fragments: theoretical results for neutron-induced fission of ${ }^{239} \mathrm{Pu}$ in good agreement with experimental data are presented in Ref. [6].

\section{Conclusions and outlook}

In the present work we have applied the Strutinsky method to the calculation of the fission barrier of ${ }^{240} \mathrm{Pu}$ and we have worked out the low-energy collective spectrum at deformations corresponding to wells and humps of the barrier. Theoretical spectra in the ground-state and isomeric well are in good agreement with experimental data. Spectra built on the humps are compatible with the experimental cross section for neutron-induced fission of ${ }^{239} \mathrm{Pu}$ at the price of adjusting theoretical barrier heights, which were calculated on the assumption of axial symmetry of the fissioning nucleus at all deformations. An improved version of the model allowing for triaxial equilibrium shapes is under study. Combining the DNS model with the scissionpoint model, we have computed mass distributions and angular anisotropies of fission fragments in neutron-induced fission of ${ }^{239} \mathrm{Pu}$ : the former are in good agreement with experiments, the latter are to be improved by including the effects of non-collective states and second-chance fission.

\section{Acknowledgement}

We are grateful to Prof. R. V. Jolos for valuable discussions.

\section{References}

1. P. Möller, et al., Phys. Rev. C 79, 064304 (2009)

2. S. Goriely, et al., Phys. Rev. C 83, 034601 (2011)

3. H. Abusara, et al., Phys. Rev. C 82, 044303 (2010)

4. Z. P. Li, et al., Phys. Rev. C 81, 064321 (2010)

5. T.M. Shneidman, et al., Phys.Rev. C 67, 014313 (2003)

6. A. V. Andreev, et al., Eur. Phys. J. A 22, 51 (2004)

7. J. Maruhn and W. Greiner, Z. Physik 251, 431 (1972)

8. G. G. Adamian, et al., Int. J. Mod. Phys. E 5, 191 (1996)

9. A. Bohr and B. Mottelson, Nuclear Structure, (W.A. Benjamin, New York, Amsterdam, 1974)

10. T. M. Shneidman, et al., Nucl. Phys. A 671, 119 (2000)

11. T. M. Shneidman, G.G. Adamian, N.V. Antonenko, R.V. Jolos, W. Scheid, Proc. of the 29th Int. Workshop on Nucl. Theory, p. 145 (Heron Press, Sofia, 2010)

12. P.G. Thirolf and D. Habs, Prog. Part. Nucl. Phys. 49, 325 (2002)

13. M. Sin, et al., Phys. Rev. C 74, 014608 (2006)

14. M. Herman, et al., Nucl. Data Sheets 108, 2655 (2007)

15. H. C. Britt, et al., Nucl. Sci. Eng. 72, 222 (1979)

16. B. I. Fursov, et al., Nuclear Data for Science and Technology, vol. I, p. 488, Trieste, 1997 (Compositori, Bologna, 1997)

17. R. Kari and S. Cierjacks, Report KFK-273 (KernForschungsZentrum, Karlsruhe, 1978)

18. P. W. Lisowski, et al., Nuclear Data for Science and Technology, p. 97 (Mito, 1988)

19. O. Shcherbakov, et al., J. Nucl. Sci. Technol. Suppl. 2, 230 (2002)

20. F. Tovesson and T. S. Hill, Nucl. Sci. Eng. 165, 224 (2010)

21. S. Bjørnholm and J. E. Lynn, Rev. Mod. Phys. 52, 725 (1980)

22. J. E. Simmons and R. L. Henkel, Phys. Rev. 120, 198

(1965); R. B. Leachman and L. Blumberg, Phys. Rev. 137, B814 (1965)

23. C. Wagemans, et al., Phys. Rev. C 30, 218 (1984) 